\title{
Brighter days ahead for Comet Austin
}

Comet Austin, now 0.75 astronomical units (AU) from the Sun and 1.5 AU from the Earth, shows two tails in this Eurnpean Southern Observatory (ESO) photograph (a 6-minute exposure taken on 24 February from La Silla, Chile). The long tail, which extends across more than 2 degrees of the sky, is a trail of ionic material evaporated from the comet's icy surface by the Sun's heat and swept back by the solar wind; the wiggles are caused by solar-wind variations and the interplanetary magnetic field. The second, much shorter tail is a trail of dust particles reflecting sunlight.

Comet Austin will be at its closest to the Sun in April. Its brightness over the coming weeks is unpredictable, depending on the amount of evaporated cometary material - mostly ice and dust - that is available to

formation of the $3^{\prime}$ CCA end, which forms a hairpin. There are also variations in the anticodon loop and stem, where the U35 central base of the anticodon is stacked underneath A37, and bases C34 and G36 are unstacked to project outwards. These conformational differences are probably induced and stabilized by interaction with the enzyme, so recognition could well follow an induced fit mechanism. Assuming that the structure of free tRNA ${ }^{\text {Gln }}$ is typical of other known tRNAs, this first description of a complexed tRNA provides another demonstration of the flexibility of these adaptor molecules. It would be interesting to analyse the uncomplexed synthetase to see whether comparably large conformational changes occur at the protein level.

Sequences important in the recognition of tRNA have been mapped in vivo and in vitro in molecular genetic experiments: it turns out that a limited number of nucleotides are responsible for the specific recognition of a tRNA by its cognate synthetase $^{5}$. The significance of a small number of key base pairs at the end of the form an extended 'coma' of reflecting material. This observation of the tail, along with some recent spectroscopic data, indicate that many species of gaseous ions are beginning to boil off. But the brightness of Comet Austin is presently falling a little behind the most optimistic predictions, which would have made it as bright as any star in the sky near its perihelion (the point of its orbit where it is closest to the Sun). From the Northern Hemisphere, the comet will appear brightest in late April and May, after it has gone round the Sun and is on its way towards Earth.

The predictions of Comet Austin's progress are made more difficult because analysis of its orbit indicates that it is a 'new' comet, approaching the Sun for the first time. The standard theory has it that a

acceptor stem is well documented: for example, the role of the pairing between the bases at positions 3 and 70 is emphasized in the $E$. coli alanine system, where any alteration affects specificity ${ }^{6}$. One surprising feature of the molecular interactions between tRNA ${ }^{\mathrm{Gln}}$ and its synthetase is that there are a very large number of them. They extend along one side of the L-shaped tRNA in a way that has been suggested by Rich and Schimmel ${ }^{7}$. Three major recognition elements can be identified. First, three polar amino-acid side chains and a buried water molecule interact with the base pairs at positions 3 and 70 , and 2 and 71 in the minor groove. The second determinant is the anticodon loop. The third results from the ability of nucleotide $\mathrm{G} 73$, the "discriminator base", to assume an unusual conformation by hydrogen-bonding to the phosphate of A72 via a hairpin turn. All these recognition elements are sequence-specific. Surface complementarity associated with hydrogen bonding is also likely to be a major discriminating factor.

What about a general recognition code, large reservoir of comets, the Oort cloud, orbits the Sun at some ten thousand AU, and that from time to time gravitational perturbations or collisions send new comets on highly elliptical or hyperbolic orbits towards the inner Solar System, where they may or may not be gravitationally captured. Periodic comets, such as Halley, have approached the Sun many times, and their behaviour at each pass is fairly consistent. But a new comet such as Austin is being heated by the Sun for the first time, and its brightness depends on the quantity of volatile materials that can be boiled off its pristine surface to form the visible coma. The current prediction from ESO is that Austin will reach a peak brightness around magnitude zero, brighter than all but a few stars.

David Lindley

the so-called second genetic code? The idea of a simple recognition code arising from direct tRNA interaction with its corresponding amino acid is discarded by Rould and his collaborators because no such direct interactions are found. They point out that the complexity of the protein-RNA association precludes the concept of a code in any conventional sense. One can speculate that the common determinant (namely, the end of the acceptor stem) might be recognized differently in the various systems. Even in the absence of sequence-specific structures (such as the G73-dependent hairpin loop), there are alternative solutions to the recognition problem. In the GlntRNA synthetase complex, recognition of the CCA stem proceeds through the minor groove of the tRNA, but this is not an absolute requirement because the major groove side is also accessible at the end of the double-helical stem. These observations could explain why the aminoacyl-tRNA synthetase family is so heterogeneous and why structural homologies are so limited. The origin of these 